\author{
C. Jackowski - E. Aghayev - M. Sonnenschein • \\ R. Dirnhofer $\cdot$ M. J. Thali
}

\title{
Maximum intensity projection of cranial computed tomography data for dental identification
}

Received: 10 May 2005 / Accepted: 3 August 2005 / Published online: 20 October 2005

C) Springer-Verlag 2005

\begin{abstract}
Dental radiographs play the major role in the identification of victims in mass casualties besides DNA. Under circumstances such as those caused by the recent tsunami in Asia, it is nearly impossible to document the entire dentition using conventional $\mathrm{x}$-rays as it would be too time consuming. Multislice computed tomography can be used to scan the dentition of a deceased within minutes, and the postprocessing software allows visualization of the data adapted to every possible antemortem x-ray for identification. We introduce the maximum intensity projection of cranial computed tomography data for the purpose of dental identification exemplarily in a case of a burned corpse. As transportable CT scanners already exist, these could be used to support the disaster victim identification teams in the field.
\end{abstract}

Keywords Forensic science $\cdot$ Forensic radiology $\cdot$ Dental identification - Maximum intensity projection (MIP) . Computed tomography

\section{Introduction}

Dental radiographs play a major role in the identification of victims in mass casualties [2] such as the recent tsunami in Asia. Without having any antemortem data, it was impossible to perform comparisons with postmortem x-rays. Postmortem dental $\mathrm{x}$-rays were limited to only two images of the bitewings, as dental x-ray documentation of the entire dentition would be inadequately time consuming under such circumstances. Data acquisition for a comparison with

C. Jackowski $(\bowtie) \cdot$ E. Aghayev $\cdot$ R. Dirnhofer $\cdot$ M. J. Thali

Institute of Forensic Medicine,

University of Bern, Bühlstrasse 20,

3012 Bern, Switzerland

e-mail: christian.jackowski@irm.unibe.ch

Tel.: +41-31-6318412

Fax: $+41-31-6313833$

M. Sonnenschein

Department of Diagnostic Radiology,

Sonnenhof Spital AG, Buchserstrasse 30

3006 Bern, Switzerland any possible antemortem dental radiographs would be a useful aid and facilitate the work of the forensic odontologists in the field. Multislice computed tomography (MSCT) can serve as an expeditious data acquisition tool. MSCT scans covering the dentition can be performed within 1-2 min. As modern CT scanners are also available mounted in trailers, it would be possible to get one into the field with minor logistic efforts. The initial reconstructed 2D CT images are not of an increased value for the identification, but 3D data do increase the possibilities. A special dental scan software can reconstruct images comparable to antemortem panoramic dental radiographs $[11,3,9]$ The purpose of this paper is to introduce the maximum intensity projection (MIP) as a 3D reconstruction algorithm for dental identification.

\section{Material and methods}

A burned corpse from the virtopsy project $[10,13]$ was MSCT scanned as the identity of the person was unclear. MSCT was performed according to the virtopsy approach on a multi-row scanner with calculated slice thickness of $1.25 \mathrm{~mm}$. The cranial CT data in this case were used to reconstruct a 3D MIP model of the head. Visualization of the model with window settings at 2,500-3,000 HU (width) and 1,500-2,000 HU (center) allowed a comparison with different antemortem dental radiographs of suspected missing persons. Reconstruction of the data was performed on a Leonardo workstation (Siemens Medical Solutions, Erlangen, Germany).

\section{Results and discussion}

Comparison of the transparent 3D MIP model of the head including the teeth allowed a positive dental identification of the body in this case (Fig. 1). The major advantage of this technique is the possibility to adapt the direction of the postmortem dental 3D model to the antemortem $\mathrm{x}$-rays at the time of comparison. Thereby the forensic pathologist or 
Fig. 1 a MIP of postmortem CCT data of a burned corpse with window settings at 2,700 HU (w) and 1,800 (c) and an oblique image orientation adapted to the projection of the antemortem radiographs well displaying the dental work that was performed. $\mathbf{b}$ and $\mathbf{c}$ Antemortem upper and lower bitewing radiographs used for identification
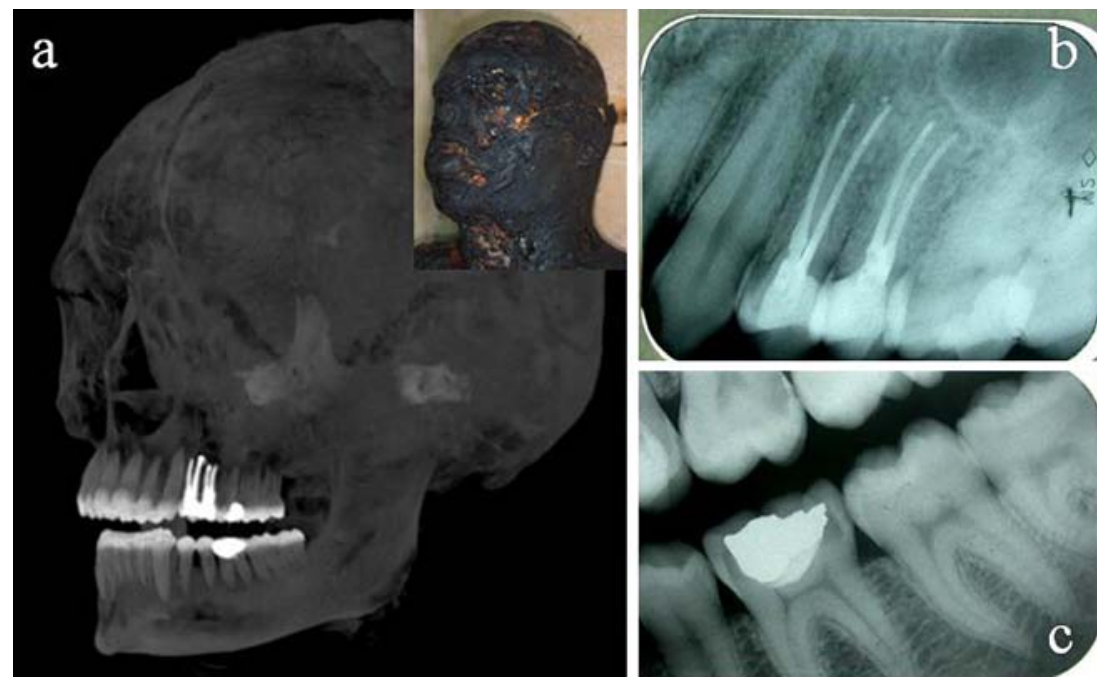

odontologist can choose exactly the same view on the postmortem model as on the antemortem radiograph, thus facilitating the matching of orofacial characteristics in general as well as the specific features of dental work with metallic or composite fillings, bridges, and crowns. Furthermore, he can assess distinctive configurations of bony structures of the jaw (maxilla and mandible), the presence and shape of teeth including the roots, the configuration of maxillary sinuses, and possible previous fractures and orhopedic procedures as longstanding pathology [1, 4-7].

A mobile CT can be installed almost everywhere, and the scanning procedure does not relevantly differ from clinical settings. For mass casualties such as the tsunami in Asia, this would allow for a detailed dental matching at a later point including the entire dentition with antemortem data that were not available at the time of postmortem dental status documentation. The data acquisition time would be decreased as there is no need to wait for any radiograph to be developed and quality-checked until the work in the field can proceed. The advantage of rapid examination time might be relativized in the field as the CT scan would surely not be limited to the head. The thorax, abdomen, and extremities contain plenty of information that might become relevant for identification and are visible within seconds on the tomogram but are missed at external inspection of putrefied corpses (e.g., coronary stents, surgical implants etc.). Furthermore, one might increase the quality of the reconstruction by adaptation of the scanning parameter to a thinner slice thickness and an increased increment for at least the cranial computed tomography (CCT), which costs another 1-2 min. A further advantage is the noninvasiveness of the documentation that makes a jaw resection dispensable. Thereby, especially the fragile teeth in burned corpses can be documented without being touched, thus avoiding the danger of destroying the dentition. The acquired data exist then in a digital form and can be sent online to any forensic odontologist within minutes.

Currently, the major disadvantage may be streak artifacts caused by the abundance of metallic dental work that can decrease the quality of $3 \mathrm{D}$ reconstructions. The detailed documentation of the shape of the restorations becomes less precise compared to classical radiographs. However, this limitation is a concern of present day fundamental CT research [12], and the metallic restorations are increasingly being replaced by more radiolucent nonmetallic composite material [8]. Furthermore, removable prostheses containing metallic material can be removed prior to scanning if the quality is intolerably decreased.

In conclusion, we are convinced that MSCT will gain importance for the identification of bodies in mass casualties as it is a rapid tool for data acquisition and offers new possibilities of comparison of antemortem and postmortem dental information.

\section{References}

1. Abrahams JJ (2001) Dental CT imaging: a look at the jaw. Radiology 219:334-345

2. Brogdon BG (1998) Forensic radiology. CRC Press LLC, Boca Raton

3. Du Chesne A, Benthaus S, Teige K et al (2000) Post-mortem orthopantomography - an aid in screening for identification purposes. Int J Legal Med 113:63-69

4. Fixott RH (2001a) How to become involved in forensic odontology. Dent Clin North Am 45:417-425

5. Fixott RH (2001b) The dental clinics of North Americaforensic odontology. Saunders, Philadelphia

6. Fixott RH, Arendt D, Chrz B et al (2001) Role of the dental team in mass fatality incidents. Dent Clin North Am 45:271292

7. Gahleitner A, Watzek G, Imhof H (2003) Dental CT: imaging technique, anatomy, and pathologic conditions of the jaws. Eur Radiol 13:366-376

8. Odlum O (2001) A method of eliminating streak artifacts from metallic dental restorations in CTs of head and neck cancer patients. Spec Care Dent 21:72-74

9. Paewinsky E, Pfeiffer H, Brinkmann B (2005) Quantification of secondary dentine formation from orthopantomograms-a contribution to forensic age estimation methods in adults. Int J Legal Med 119:27-30

10. Thali MJ, Yen K, Schweitzer W et al (2003) Virtopsy, a new imaging horizon in forensic pathology: virtual autopsy by postmortem multislice computed tomography (MSCT) and magnetic resonance imaging (MRI) - a feasibility study. J Forensic Sci 48:386-403 
11. Thali MJ, Markwalder T, Jackowski C et al (2005) Dental CT imaging as a screening tool for dental profiling: advantages and limitations. J Forensic Sci, in print

12. Watzke O, Kalender WA (2004) A pragmatic approach to metal artifact reduction in $\mathrm{CT}$ : merging of metal artifact reduced images. Eur Radiol 14:849-856
13. Yen K, Sonnenschein M, Thali MJ et al (2005) Postmortem multislice computed tomography and magnetic resonance imaging of odontoid fractures, atlantoaxial distractions and ascending medullary edema. Int J Legal Med 119:129-136 